\title{
Underlying conditions in chronic pulmonary aspergillosis including simple aspergilloma
}

\author{
N.L. Smith and D.W. Denning
}

ABSTRACT: Chronic pulmonary aspergillosis (CPA) is a condition caused by the ubiquitous fungus Aspergillus fumigatus in non-immunocompromised individuals. Numerous underlying conditions have been associated with CPA.

Details of the underlying conditions of 126 CPA patients attending our tertiary referral clinic from all over the UK were extracted from the clinical notes, and the distribution of these underlying conditions was analysed. For those with several underlying pulmonary conditions, one was nominated as the primary condition.

Many patients presented with multiple underlying conditions, and a total of 232 underlying conditions were identified for the 126 patients. Previous classical tuberculosis and nontuberculous mycobacterial infection were the most common primary underlying conditions (15.3\% and $14.9 \%$, respectively). Others included allergic bronchopulmonary aspergillosis (ABPA), chronic obstructive pulmonary condition (COPD) and/or emphysema, pneumothorax and prior treated lung cancer. Some conditions were found more often as one of multiple underlying conditions, while others were found only as secondary underlying conditions.

Tuberculosis, non-tuberculous mycobacterial infection and ABPA remain the predominant risk factors for development of CPA, with COPD, prior pneumothorax or treated lung cancer also relatively common among our referrals. Many patients have multiple underlying pulmonary conditions. CPA should be considered when upper lobe cavitary or fibrotic disease and systemic symptoms are present in those with lung disease.

KEYWORDS: Aspergilloma, chronic necrotising pulmonary aspergillosis, itraconazole, lung carcinoma, non-tuberculous mycobacteria

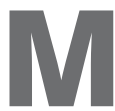
ultiple diseases are caused by Aspergillus fumigatus, an opportunistic pathogen and allergenic fungus. Estimates suggest that humans inhale several hundred spores each day [1]. In most people these are cleared without causing disease. However, in atopic individuals, A. fumigatus can cause allergic bronchopulmonary aspergillosis (ABPA). It can cause acute and subacute invasive disease in immunocompromised patients, and chronic pulmonary aspergillosis (CPA) in non-immunocompromised patients. In CPA, Aspergillus gradually destroys lung tissue, resulting in the formation and expansion of cavities. These may contain a fungal ball (aspergilloma) [2, 3]. If untreated, destruction can eventually encompass an entire lobe or lung; even with treatment, the morbidity and mortality of CPA remains high [3, 4]. Patients require longterm maintenance antifungal therapy to prevent relapse and many continue to have some disability, for example fatigue and intermittent secondary infections of remaining pulmonary cavities [4].
Prior pulmonary conditions are often evident in CPA patients $[4,5]$. Knowledge of the relative frequencies of these is important for clinicians to facilitate awareness, and for quicker disease identification and earlier treatment. Currently, many cases are diagnosed late in their course. Studies following 18-24 patients have identified multiple underlying conditions, including tuberculosis, emphysema, COPD, pneumothorax and previous thoracic surgery $[4,5]$. A population estimate of CPA numbers requires a larger experience than this. Therefore, we analysed the underlying conditions for 126 patients with CPA, including simple aspergillomas, attending our referral clinic.

\section{METHODS}

Patients attending the National Aspergillosis Centre (North West Lung Centre, University Hospital of South Manchester, Wythenshawe Hospital, Manchester, UK) from 2002 to November 23, 2009 with physician-diagnosed CPA or simple aspergilloma were analysed. The referral base has
AFFILIATIONS

The School of Translational

Medicine, University of Manchester, Manchester Academic Health Science Centre and University Hospital of South Manchester, Manchester, UK.

CORRESPONDENCE

D.W. Denning

2nd Floor Education and Research Centre

Wythenshawe Hospital

Southmoor Road

Manchester

M23 9LT

UK

E-mail: ddenning@manchester.ac.uk

Received:

April 082010

Accepted after revision:

June 232010

First published online:

July 012010 
gradually widened from the Manchester area to the Northwest of England and is currently national. The North West Lung Centre $(26,000$ attendances per yr) serves 350,000 people in the local population and 6.3 million people within the North West, who live in a disadvantaged area with a high burden of lung disease (e.g. standardised mortality ratio for COPD locally is 170).

CPA was defined as non-immunocompromised patients with the presence of: 1) at least one pulmonary cavity on thoracic imaging; 2) positive anti-aspergillus immunoglobulin $G$ antibodies (precipitins) in blood, cultures or biopsy implicating Aspergillus spp; and 3) either symptoms (usually weight loss, fatigue, cough, haemoptysis and breathlessness) for $>3$ months, with slowly progressive destruction of the lung with or without one or more fungal ball in a cavity (chronic cavitary pulmonary aspergillosis (CCPA), or chronic fibrosing pulmonary aspergillosis (CFPA)), or a fungal ball in a cavity without evidence of progression and few or no symptoms (simple aspergillomas) (table 1) [4, 6]. Immunocompromised patients (i.e. those with rheumatoid arthritis receiving oral prednisolone $>7.5 \mathrm{mg} \cdot \mathrm{day}^{-1}$ or other immunosuppression, or HIV infection with CD 4 count $<200$ cells $\cdot \mu \mathrm{L}^{-1}$ ) were excluded. Therefore, we did not include patients with sub-acute invasive aspergillosis (SAIA), also known as chronic necrotising pulmonary aspergillosis. Patients who presented with acute invasive aspergillosis and who went on to develop CPA because of incomplete resolution of invasive disease were also excluded. There were three such patients. Details of the underlying conditions were extracted from the notes of patients, and the frequency of these conditions was analysed. The underlying conditions were documented as fully as possible using details from the referring physician and, in the case of chronic obstructive pulmonary disease (COPD) and/or emphysema, both radiologically by computed tomography of the thorax and by pulmonary function tests. Where many underlying conditions were present simultaneously, a clinical decision was made (D.W. Denning) as to which was the earliest and/or predominant factor in the development of CPA. This was termed the primary underlying condition.

\section{RESULTS}

Patient demographics are shown in table 2. Most patients were white $(89.7 \%)$, and the mean age at analysis was 59 yrs 8 months $(n=109)$, although there was a wide age range (27 yrs 2 months to 86 yrs 8 months). Most patients presented with CCPA $(88.9 \%)$. Figure 1 shows four radiological examples of CCPA.

A total of 232 underlying conditions were identified for the 126 CPA cases analysed. For each case, one primary underlying condition was identified. The frequency of each disease as an underlying condition and as a primary underlying condition is listed in table 3.

Previous tuberculosis (either classical or atypical) was the most commonly identified primary underlying condition (38 (30.2\%) out of 126 cases). The relative ratio of non-tuberculous mycobacterial infection $(14.9 \%)$ to classical tuberculosis $(15.3 \%)$ was much higher than nationally reported figures. The second common, primary underlying condition was ABPA (15 (11.9\%) out of 126 cases). Other less common primary underlying conditions included COPD and/or emphysema (12 (9.5\%) out of 126 cases), a history of pneumothorax (12 (9.5\%) out of 126), prior treated lung cancer $(12(9.5 \%)$ out of 126$)$, pneumonia (10 $(7.9 \%)$ out of 126$)$, rheumatoid arthritis without immunocompromise (four (3.2\%) out of 126) and severe asthma with fungal sensitisation (SAFS; two (1.6\%) out of 126$)$.

\section{TABLE 1 Glossary of terms}

Aspergilloma

Simple aspergilloma

Chronic cavitary pulmonary aspergillosis

Chronic fibrosing pulmonary aspergillosis

Subacute invasive aspergillosis or chronic necrotising pulmonary aspergillosis

Acute invasive aspergillosis
An approximately spherical shadow with surrounding air, also called a fungal ball, in a pulmonary cavity, with serological or microbiological evidence that Aspergillus spp. is present in the material. This is a radiological or morphological description, not a disease descriptor

Single pulmonary cavity containing a fungal ball, with serological or microbiological evidence implicating Aspergillus spp. in a non-immunocompromised patient with minor or no symptoms and no radiological progression over at least 3 months of observation One or more pulmonary cavities which may or may not contain a fungal ball, with serological or microbiological evidence implicating Aspergillus spp. in a non-immunocompromised patient (or one whose immunocompromising condition has remitted or is trivial) with significant pulmonary or systemic symptoms and overt radiological progression (new cavities, increasing pericavity infiltrates or increasing fibrosis) over at least 3 months of observation. If biopsy of the affected area is performed, it demonstrates hyphae with surrounding chronic inflammation and fibrosis but not tissue invasion

Severe fibrotic destruction of at least two lobes of lung complicating chronic cavitary pulmonary aspergillosis leading to a major loss of lung function. Usually the fibrosis is in the form of consolidation, but it may be large cavities with surrounding fibrosis. Severe fibrotic destruction of one lobe with a cavity is simply referred to as chronic cavitary pulmonary aspergillosis affecting that lobe Invasive aspergillosis, usually in mildly immunocompromised patients, occurring over 1-3 months, with marked pleitrophic radiological features (cavitation, nodules and progressive consolidation with "abscess formation"), with hyphae visible in destroyed lung tissue or inferred from microbiological investigations (i.e. positive Aspergillus antigen) and pace of disease Invasive aspergillosis, usually in markedly immunocompromised patients, occurring over $<1$ month. May be angioinvasive or not. Radiological features include nodules or progressive consolidation often with a "halo" sign inferring angioinvasive disease, cavity (or air crescent) formation, pleural effusion and miliary appearance (if associated with massive spore exposure). If biopsy is performed, hyphae are visible in destroyed lung tissue. Most diagnoses inferred from typical radiological appearances on computed tomography scanning, microbiological investigations (i.e. positive Aspergillus antigen), immunocompromised status and rapid pace of disease 


\begin{tabular}{|c|c|c|}
\hline TABLE 2 & \multicolumn{2}{|c|}{$\begin{array}{l}\text { Patient demographics and manifestation of } \\
\text { pulmonary aspergillosis }\end{array}$} \\
\hline Subjects $n$ & & $126(100)$ \\
\hline Alive & & $109(86.5)$ \\
\hline Deceased & & $17(13.5)$ \\
\hline \multicolumn{3}{|l|}{ Age } \\
\hline Alive & & $\begin{array}{c}59 \text { yrs } 8 \text { months } \\
\text { (27 yrs } 2 \text { months- } 86 \text { yrs } 8 \text { months) }\end{array}$ \\
\hline \multicolumn{2}{|c|}{ Deceased, age at death } & $\begin{array}{c}63 \text { yrs } 10 \text { months } \\
\text { (49 yrs } 7 \text { months }-75 \text { yrs } 5 \text { months) }\end{array}$ \\
\hline \multicolumn{3}{|r|}{ The } \\
\hline Male & & $75(59.5)$ \\
\hline Female & & $51(40.5)$ \\
\hline \multicolumn{3}{|l|}{ Ethnicity } \\
\hline \multicolumn{2}{|c|}{ White British or White Irish } & $111(88.1)$ \\
\hline \multicolumn{2}{|c|}{ White Danish } & $1(0.8)$ \\
\hline \multicolumn{2}{|c|}{ Any other White background } & $1(0.8)$ \\
\hline \multicolumn{2}{|c|}{$\begin{array}{l}\text { Asian/Asian British (Indian), Indian } \\
\text { or Gujarti }\end{array}$} & $4(3.2)$ \\
\hline \multicolumn{2}{|c|}{$\begin{array}{l}\text { Black/Black British (African), } \\
\text { Zimbabwean or Kenyan }\end{array}$} & $3(2.4)$ \\
\hline \multicolumn{2}{|c|}{ Black (Caribbean) } & $1(0.8)$ \\
\hline \multicolumn{2}{|l|}{ Jamaican } & $1(0.8)$ \\
\hline \multicolumn{2}{|l|}{ Unknown } & $4(3.2)$ \\
\hline \multicolumn{3}{|l|}{ Disease } \\
\hline \multicolumn{2}{|c|}{ Simple aspergilloma } & $10(7.9)$ \\
\hline \multicolumn{2}{|c|}{ CCPA \pm aspergilloma } & $112(88.9)$ \\
\hline \multicolumn{2}{|c|}{ CCPA+CFPA } & $4(3.2)$ \\
\hline
\end{tabular}

Data are presented as $n$ (\%) or mean (range). CCPA: chronic cavitary pulmonary aspergillosis; CFPA: chronic fibrosing pulmonary aspergillosis.

Of all the 232 underlying conditions identified for $126 \mathrm{CPA}$ cases, COPD/emphysema was the most common (42 (33.3\%) out of 126). Classical tuberculosis and non-tuberculous mycobacterial infection were also very common (41 (32.5\%) out of $126)$, as were pneumothorax (21 (16.7\%) out of 126), pneumonia (28 $(22.2 \%)$ out of 126$)$ and asthma (13 (10.3\%) out of 126$)$.

The underlying condition distribution for simple aspergilloma $(n=10)$ was similar. Tuberculosis (classical and atypical) again accounted for $30 \%$ of cases (three out of 10). Pneumonia was identified as the primary underlying condition in $20 \%$ of cases (two out of 10), while pneumothorax, bullae, ABPA, sarcoidosis and previously cured lung cancer were each identified as the primary underlying condition in one patient each $(10 \%)$. In addition to these, thoracic surgery, SAFS, asthma and bullae were each identified as secondary underlying conditions.

17 patients were deceased at the time of analysis. The mean age at death was 63 yrs 10 months. In eight patients the cause of death was not ascertained as they did not die in hospital. For the causes of death that were ascertained, pneumonia was the most common cause of death (five (55.5\%) out of nine), complicated by acute respiratory distress syndrome in one patient. One patient each died of severe sepsis without pneumonia, cardiac arrest, lung cancer and respiratory failure.

\section{DISCUSSION}

Analysis of the underlying conditions was complicated as many patients presented with multiple underlying conditions at the time of diagnosis with CPA. 232 underlying conditions were identified in 126 analysed cases of CPA, resulting in a mean (range) of 1.8 (1-4) underlying conditions per CPA case. For cases of simple aspergilloma in particular, a mean (range) of $1.6(1-3)$ conditions per case was identified.

Other reports have also identified multiple underlying conditions for CPA cases [3, 4]. DeNNING et al. [4] identified a mean (range) of 2.05 (1-3) conditions per CPA case, while CAMUSET et al. [5] identified a mean of 1.7 conditions per patient. The study by DENNING et al. [4] included patients with CCPA, SAIA and aspergilloma while the study by CAMUSET et al. [5] included patients with CCPA and SAIA. Our study includes patients with: CCPA; CCPA and CFPA; and simple aspergillomas. The reader should remain aware that although both previous studies listed all the underlying conditions identified for the patients, no attempt was made to identify which was the primary underlying condition. In our study, we similarly identified all the underlying conditions for each case of CPA. However, an effort was also made to determine which was the most important, the primary cause of the CPA and, although in most instances this was apparently straightforward based on the temporal sequence and severity of prior condition, this categorisation should be regarded as intrinsically arbitrary.

\section{Tuberculosis}

As with previous studies, we found that both non-tuberculous and classical tuberculosis were very common, being identified as an underlying condition in $32.5 \%$ (41 out of 126) of patients [3-13]. Where it was identified it was usually designated as the primary underlying condition (38 out of 41 tuberculosis cases). Thus, tuberculosis was the most common primary underlying condition, accounting for 38 (30.2\%) out of 126 of the CPA cases.

Our finding is lower than in previous studies, which identified tuberculosis as an underlying condition in $50-72 \%$ of CPA patients $[4,5,10]$. This difference is probably due to the fact that these studies only analysed 18-25 patients, while we analysed 126 patients. The difference could also reflect a decreasing prevalence of tuberculosis, as our study finished more recently than the other studies. Larger studies have been carried out to investigate aspergillomas in particular. A UK study found tuberculosis (non-tuberculous or classical) to be an underlying condition in $31.2 \%$ (27 out of 85 ) of patients, a result that is similar to ours [7]. This supports the idea that the differences seen in the other studies are due to the small sample sizes used, or specialised referral patterns. Studies of aspergillomas in Taiwan, South Korea and India identified tuberculosis as the aetiological factor in up to $93 \%$ of cases of CPA $[8,9,14]$. This high level of tuberculosis as an underlying condition probably reflects the fact that tuberculosis is more prevalent in India and Taiwan than in the UK.

The importance of tuberculosis in the development of aspergillosis is supported by the results from studies of tuberculosis patients. These studies have found that out of 544 patients who were left with a residual cavity $\geqslant 2.5 \mathrm{~cm} 1 \mathrm{yr}$ after cured tuberculosis, 36\% had positive Aspergillus antibodies and 22\% had radiological aspergillomas after 3 yrs [11, 12]. At the time 
that these reports were published, CCPA without a fungal ball present on chest radiograph was not separated from aspergilloma, so these reports probably contain cases of what we now recognise as CCPA, SAIA and CFPA, as well as simple aspergilloma cases. Given that $21-35 \%$ of patients who survive pulmonary tuberculosis have residual cavities $[15,16]$ it implies that $8-12 \%$ patients who recover from classical tuberculosis develop CPA over 4 yrs. However, this figure
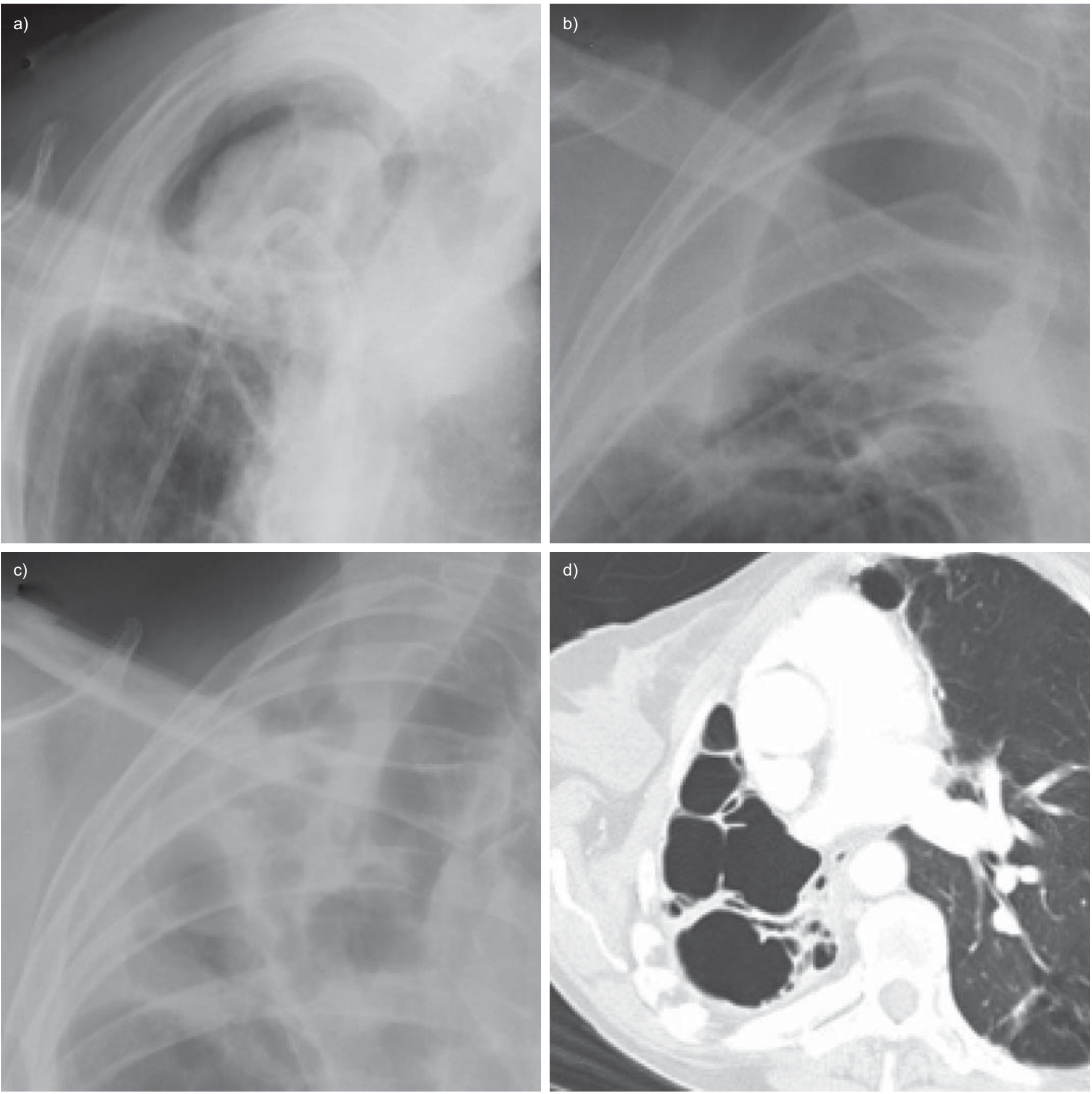

FIGURE 1. Radiological examples of chronic cavitary pulmonary aspergillosis. a) A typical fungal ball in a cavity related to idiopathic kyphoscoliosis which presented with life-threatening haemoptysis. b) The left apex with marked pleural thickening, a single large cavity without a fungal ball, which presented several years after a spontaneous pneumothorax with marked coughing and severe fatigue. c) Multiple cavities without any evidence of a fungal ball in a patient who had suffered from multiple chest infections during her life, resulting in an unsuccessful partial right lower lobectomy 45 yrs earlier. d) Multiple empty cavities completely replacing the right lung with remarkably little pleural thickening on a computed tomography image in an African-Asian female who had tuberculosis $>10$ yrs earlier and presented with weight loss and breathlessness. 
may be excessively high for the UK as clinical experience in the management of pulmonary tuberculosis would estimate the rate of residual cavities to be lower. One study identified aspergillus co-infection in $14(10 \%)$ out of 136 cases of Mycobacterium xenopi pulmonary infection [17], while another found that aspergillosis developed in $4 \%$ of 302 patients with M. kansasii infections [18].

Of note, the proportion of non-tuberculous mycobacterial infection and classical tuberculosis found in our study is very different from the relative frequencies of these entities in the UK, with a much higher frequency of non-tuberculous mycobacterial infection being found in our patients. This has also been found in previous studies [4,5].

\section{ABPA, SAFS and asthma}

After tuberculosis, the second most common primary underlying condition was ABPA. This was identified as an underlying condition in $18(14.3 \%)$ out of 126 CPA cases. In 15 $(11.9 \%)$ out of $126 \mathrm{CPA}$ cases it was the primary underlying condition, highlighting its importance. Case reports of coexistent ABPA and CPA have been documented and one study of aspergillomas identified ABPA as an underlying condition in $11.8 \%$ (10 out of 85$)$ of the cases reviewed $[3,7,18,19]$, which is similar to our findings.

SAFS was identified as an underlying condition in three $(2.4 \%)$ out of 126 of our patients, and was found to be the primary underlying condition for $1.6 \%$ (two out of 126 ) of

TABLE 3 Underlying conditions identified in analysed chronic pulmonary aspergillosis (CPA) cases and comparison to literature

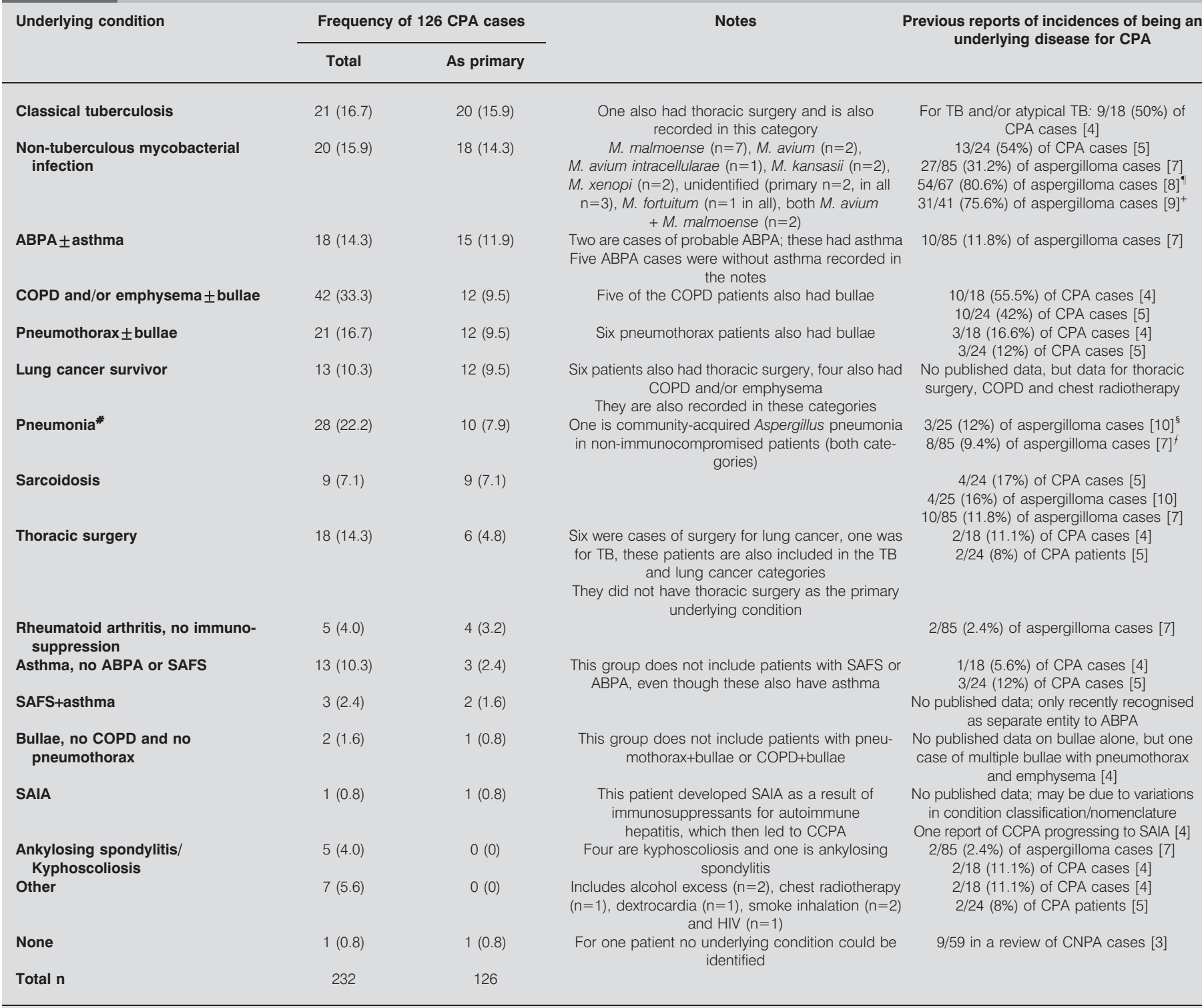

Data are presented as $n(\%)$, unless otherwise stated. ABPA: allergic bronchopulmonary aspergillosis; COPD: chronic obstructive pulmonary disease; SAFS: severe asthma with fungal sensitisation; SAIA: subacute invasive aspergillosis; TB: tuberculosis; CCPA: chronic cavitary pulmonary aspergillosis; CNPA: chronic necrotising pulmonary aspergillosis.

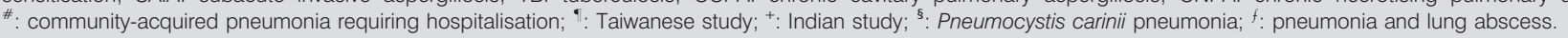


the CPA cases [20]. This has not been reported previously, but this is probably due to the fact that it has only recently been recognised as a subset of severe asthma separate from ABPA.

Asthma, without ABPA or SAFS, was identified in $10.3 \%$ (13 out of 126) of the patients. It was identified as the primary underlying condition in $2.4 \%$ (three out of 126) of CPA cases. Asthma has been previously identified in $5.6-12 \%$ of CPA patients [3-5]. The fact that it is a relatively common underlying condition but a rare primary underlying condition suggests that, rather than the presence of asthma itself, it is the interaction of the asthma with other lung conditions that predisposes patients to CPA.

\section{COPD and/or emphysema}

For this study, COPD and emphysema were classed as the same condition, thus patients whose notes recorded both were only classified as having COPD and/or emphysema once. Overall, COPD and/or emphysema were the most common underlying condition, identified in one third of patients (42 (33.3\%) out of 126). Five of these patients also had bullae. COPD and/or emphysema have been previously identified as underlying conditions in $42-66.9 \%$ of CPA patients [3-5], which is similar to our results.

As well as being a common underlying condition, COPD/ emphysema was identified as a primary underlying condition in $9.5 \%$ (12 out of 126 ) of the CPA patients analysed. COPD and emphysema are very common lung conditions, and as such it is not surprising that they are found in a large proportion of the CPA patients. However, the fact that they were identified as primary underlying conditions demonstrates that they are not only a co-morbidity, but actually contribute to the development of CPA. Recent data indicate a substantial increase in diagnosed acute invasive pulmonary aspergillosis (IPA) in COPD, which has a 95\% mortality rate $[21,22]$. Transformation from CPA to acute IPA following an exacerbation of COPD which is treated with corticosteroids is possible.

\section{Pneumonia}

Pneumonia has been identified as a predisposing factor for aspergilloma in two previous studies. One identified Pneumocystis carinii pneumonia in $12 \%$ of aspergilloma cases, while another identified pneumonia and lung abscess in $9.4 \%$ of aspergilloma cases [7, 10]. Our study identified previous pneumonia as an underlying condition in $22.2 \%$ (28 out of 126 ) of the CPA patients analysed. In $7.9 \%$ of patients, it was found to be the primary underlying condition. Interestingly, one of these was a case of community-acquired aspergillus pneumonia in a non-immunocompromised patient.

\section{Sarcoidosis}

Sarcoidosis has been previously identified as a predisposing factor for CPA, with a number of case reports having been published [19, 23]. Cohort studies have identified sarcoidosis as an underlying condition in $11.8-17 \%$ of CPA patients $[5,7,10]$; these studies involved 24-85 patients, with the study of 85 patients reporting $11.8 \%$. The fact that the other studies are higher is probably due to the small patient numbers. In our study, nine $(7.1 \%)$ patients presented with sarcoidosis as an underlying condition. In all nine patients sarcoidosis was identified as the primary underlying condition. This suggests that while not the most common underlying condition, where sarcoidosis is present it is the main reason for the development of CPA.

The importance of sarcoidosis in the development of CPA has been identified previously. A study that followed 100 sarcoidosis patients over a $10-y r$ period found that $10 \%$ developed aspergillomas; a partial estimate of CPA cases as not all will develop fungus balls [24]. The overall incidence rate per 100,000 person-yrs is estimated at 5 for sarcoidosis, suggesting that 3,000 new cases are diagnosed each year in the UK [25]. If $10 \%$ of these develop aspergilloma then we should expect at least 300 new aspergilloma cases each year in this population.

\section{Pneumothorax}

Pneumothorax ( \pm bullae) was identified in $16.7 \%$ (21 out of 126$)$ of our CPA patients. Six of these patients had pneumothorax plus bullae, while the remainder had pneumothorax without bullae. In $9.5 \%$ (12 out of 126) of patients, pneumothorax was found to be the primary underlying condition. History of pneumothorax has been identified previously in $11.1-12 \%$ of CPA cases, of which one case also had multiple bullae $[4,5]$. The numbers of patients in these studies are much lower than in ours, but they demonstrate that pneumothorax is an important predisposing factor for CPA.

\section{Lung cancer and thoracic surgery}

Prior treated lung cancer was identified in 13 (10.3\%) out of 126 of the analysed CPA cases. Of these, 12 were cases where it was the primary underlying condition. This is likely to partly be a result of the treatment, which may include chemotherapy, chest radiotherapy and/or thoracic surgery. Chemotherapy, chest radiography and thoracic surgery have been identified as predisposing factors for CPA previously [3-5]. In addition, COPD is a common co-existing condition in those with lung cancer and was common, but not universal, in our patients. We recognise that this is a somewhat arbitrary choice of underlying disease but we feel it reflects burden of disease more than a simple COPD label.

In our study, thoracic surgery was identified as an underlying condition in $14.3 \%$ (18 out of 126 ) of patients. This includes six patients who had surgery for lung cancer, and one who had surgery for TB. These patients are included in both groups. Thoracic surgery was identified as the primary underlying condition in six $(4.8 \%)$ patients, demonstrating its importance as a predisposing factor for CPA. Again this is an arbitrary choice as the primary choice of underlying disease, however, given the nature of the cases we feel it is appropriate.

\section{Rheumatoid arthritis}

Rheumatoid arthritis (RA) was identified as an underlying condition in $4.0 \%$ (five out of 126) of our CPA patients, and as a primary underlying condition in $3.2 \%$ (four out of 126). We care for more than 10 patients with RA and pulmonary aspergillosis and only included those receiving little or no immunosuppressive treatment (i.e. $\leqslant 7.5 \mathrm{mg}$ prednisolone daily).

Cases of CPA following RA have been reported previously, although some also have other predisposing factors, such as 
pneumothoraces or tuberculosis [3, 13]. JEWKES et al. [7] identified upper lobe fibrosis and/or cavitation associated with RA as underlying $2.4 \%$ (two out of 85 ) of their aspergilloma cases.

\section{Other conditions}

Some conditions were identified as one of multiple underlying conditions for any CPA case, but not as primary underlying conditions. These include ankylosing spondylitis, kyphoscoliosis, alcohol excess, bullae (without pneumothorax or COPD), chest radiotherapy, smoke inhalation and HIV. All of these have been previously identified as increasing CPA predisposition $[3-5,6,10]$. One patient was recorded as having SAIA as the primary underlying condition. This patient developed SAIA as a result of immunosuppressive therapy for autoimmune hepatitis, which then led to CCPA when her immunosuppression was withdrawn. For one patient no underlying condition could be identified. Other studies have also reported cases where no underlying disease could be identified [3].

\section{General comments on pathogenesis of CPA}

The results discussed here demonstrate the importance of underlying conditions in the development of CPA. Many of the underlying conditions discussed have effects on the physical structure of the lung. COPD was the most common underlying condition overall, and it may be that bullous emphysema is the primary driver for the development of CPA in this population. Pneumothoraces, lung cancer and thoracic surgery, by their nature and by their treatment, result in lung damage, while tuberculosis can leave cavities in the lung $[11,12,15]$. Pneumonia can cause extensive damage and scarring, RA can lead to the development of pulmonary fibrosis and sarcoidosis, particularly the late stage fibrotic form, results in lung fibrosis and cavities $[7,24]$. ABPA can also result in lung fibrosis [26]. All of these result in areas of damaged lung which Aspergillus can colonise and infect. Once localised in the damaged lung, the fungus can grow and either form a simple fungal ball or go on to invade the lung parenchyma and cause/expand cavities. Other identified conditions have a strong fungal component themselves. These include ABPA and SAFS, and the presence of the fungus in these patients may precipitate colonisation and development of CPA. Genetic factors may be important in the development of CPA, and some have been identified but this work is in its infancy, as it is for most respiratory conditions [27-29].

\section{SUPPORT STATEMENT}

This study was funded by the UK Medical Research Council and infrastructure provided by the NHS National Institute of Health Research Translational Research Facility in Respiratory Medicine.

\section{STATEMENT OF INTEREST}

A statement of interest for D.W. Denning can be found at www.erj. ersjournals.com/site/misc/statements.xhtml

\section{ACKNOWLEDGEMENTS}

We would like to thank C. Harris (University of Manchester, Manchester, UK) for help obtaining the patients' notes required for this study.

\section{REFERENCES}

1 Latge JP. Aspergillus fumigatus and aspergillosis. Clin Microbiol Rev 1999; 12: 310-350.

2 Soubani AO, Chandrasekar PH. The clinical spectrum of pulmonary aspergillosis. Chest 2002; 121: 1988-1999.

3 Saraceno JL, Phelps DT, Ferro TJ, et al. Chronic necrotizing pulmonary aspergillosis: approach to management. Chest 1997; 112: $541-548$.

4 Denning DW, Riniotis K, Dobrashian R, et al. Chronic cavitary and fibrosing pulmonary and pleural aspergillosis: case series, proposed nomenclature change, and review. Clin Infect Dis 2003; 37: Suppl. 3, S265-S280.

5 Camuset J, Nunes H, Dombret MC, et al. Treatment of chronic pulmonary aspergillosis by voriconazole in nonimmunocompromised patients. Chest 2007; 131: 1435-1441.

6 Hope WW, Walsh TJ, Dennind DW. The invasive and saprophytic syndromes due to Aspergillus spp. Med Mycol 2005; 43: Suppl. 1, S207-S238.

7 Jewkes J, Kay PH, Paneth M, et al. Pulmonary aspergilloma: analysis of prognosis in relation to haemoptysis and survey of treatment. Thorax 1983; 38: 572-578.

8 Chen JC, Chang YL, Luh SP, et al. Surgical treatment for pulmonary aspergilloma: a 28 year experience. Thorax 1997; 52: 810-813.

9 Shah R, Vaideeswar P, Pandit SP. Pathology of pulmonary aspergillomas. Indian J Pathol Microbiol 2008; 51: 342-345.

10 Addrizzo-Harris DJ, Harkin TJ, McGuinness G, et al. Pulmonary aspergilloma and AIDS. A comparison of HIV-infected and HIVnegative individuals. Chest 1997; 111: 612-618.

11 Aspergillus in persistent lung cavities after tuberculosis. A report from the Research Committee of the British Tuberculosis Association. Tubercle 1968; 49: 1-11.

12 Aspergilloma and residual tuberculous cavities - the results of a resurvey. Tubercle 1970; 51: 227-245.

13 Sugino K, Hasegawa C, Sano G, et al. Pathophysiological study of chronic necrotizing pulmonary aspergillosis. Jpn J Infect Dis 2008; 61: 450-453.

14 Nam HS, Jeon $\mathrm{K}$, Um SW, et al. Clinical characteristics and treatment outcomes of chronic necrotizing pulmonary aspergillosis: a review of 43 cases. Int J Infect Dis 2010; 14: e479-e482.

15 Sonnenberg P, Murray J, Glynn JR, et al. HIV-1 and recurrence, relapse, and reinfection of tuberculosis after cure: a cohort study in South African mineworkers. Lancet 2001; 358: 1687-1693.

16 Andréjak C, Lescure FX, Pukenyte E, et al. Mycobacterium xenopi pulmonary infections: a multicentric retrospective study of 136 cases in north-east France. Thorax 2009; 64: 291-296.

17 Maliwan N, Zvetina JR. Clinical features and follow up of 302 patients with Mycobacterium kansasii pulmonary infection: a 50 year experience. Postgrad Med J 2005; 81: 530-533.

18 Shah A, Khan ZU, Chaturvedi S, et al. Allergic bronchopulmonary aspergillosis with coexistent aspergilloma: a long-term followup. I Asthma 1989; 26: 109-115.

19 Israel RH, Poe RH, Bomba PA, et al. The rapid development of an aspergilloma secondary to allergic bronchopulmonary aspergillosis. Am J Med Sci 1980; 280: 41-44.

20 Denning DW, O'Driscoll BR, Hogaboam CM, et al. The link between fungi and severe asthma: a summary of the evidence. Eur Respir J 2006; 27: 615-626.

21 Bulpa P, Dive A, Sibille Y. Invasive pulmonary aspergillosis in patients with chronic obstructive pulmonary disease. Eur Respir J 2007; 30: 782-800.

22 Guinea J, Torres-Narbona M, Gijón P, et al. Pulmonary aspergillosis in patients with chronic obstructive pulmonary disease: incidence, risk factors, and outcome. Clin Microbiol Infect 2010; 16: 870-877.

23 Panjabi C, Sahay S, Shah A. Aspergilloma formation in cavitary sarcoidosis. J Bras Pneumol 2009; 35: 480-483. 
24 Wollschlager C, Khan F. Aspergillomas complicating sarcoidosis. A prospective study in 100 patients. Chest 1984; 86: 585-588.

25 Lee TM, Greenberger PA, Patterson R, et al. Stage V (fibrotic) allergic bronchopulmonary aspergillosis. A review of 17 cases followed from diagnosis. Arch Intern Med 1987; 147: 319-323.

26 Carvalho A, Pasqualotto AC, Pitzurra L, et al. Polymorphisms in Toll-like receptor genes and susceptibility to pulmonary aspergillosis. J Infect Dis 2008; 197: 618-621.

27 Vaid M, Kaur S, Sambatakou H, et al. Distinct alleles of mannosebinding lectin (MBL) and surfactant proteins A (SP-A) in patients with chronic cavitary pulmonary aspergillosis and allergic bronchopulmonary aspergillosis. Clin Chem Lab Med 2007; 45: 183-186.

28 Sambatakou H, Pravica V, Hutchinson IV, et al. Cytokine profiling of pulmonary aspergillosis. Int J Immunogenet 2006; 33: 297-302.

29 Crosdale DJ, Poulton KV, Ollier WE, et al. Mannose-binding lectin gene polymorphisms as a susceptibility factor for chronic necrotizing pulmonary aspergillosis. J Infect Dis 2001; 184: 653-656. 